\title{
El apixaban fue efectivo en comparación con placebo en el tratamiento extendido del tromboembolismo venoso
}

Apixaban was effective when compared to placebo for extended treatment of venous thromboembolism

Agnelli G. N Engl J Med 2013;368:699-708

\section{Objetivo}

Comparar el uso de apixaban en dos diferentes dosis frente a placebo para el tratamiento extendido del tromboembolismo venoso.

\section{Diseño y lugar}

Ensayo clínico controlado y aleatorizado (ECCA), doble ciego. Fue realizado en 328 centros en 28 países, entre mayo de 2008 y julio de 2011.

\section{Pacientes}

Se eligieron pacientes a partir de 18 o más años de edad; con trombosis venosa profunda (TVP) sintomática o tromboembolismo pulmonar (TEP, con o sin TVP), objetivamente confirmados; que hubieran sido tratados por 6 a 12 meses con tratamiento anticoagulante estándar, o hubieran completado tratamiento con apixaban o enoxaparina y warfarina como participantes del ECCA AMPLIFY; que no hubieran tenido recurrencia síntomática durante el tratamiento anticoagulante inicial; y que presentaran discrepancias entre el equipo tratante sobre la decisión de continuar o suspender la anticoagulación.

\section{Intervención}

Se aleatorizaron 2.486 pacientes, con relación 1:1:1, a recibir apixaban $2,5 \mathrm{mg}$, apixaban $5 \mathrm{mg}$, o placebo, vía oral, dos veces al día, durante 12 meses.

\section{Medición de resultados principales}

El análisis se realizó por intención de tratar sobre 2.482 individuos. Se estableció como resultado principal combinado la recurrencia de tromboembolismo venoso (TEV, el cual incluyó eventos fatales y no fatales de TEP y eventos de TVP) y muerte por todas las causas. Como resultado secundario se estableció la recurrencia de TEV y muerte relacionada a TEV.

El resultado principal de seguridad fue la ocurrencia de eventos hemorrágicos mayores (caída de $\geq 2 \mathrm{~g} / \mathrm{dl}$ hemoglobina, transfusión de $\geq 2$ unidades de glóbulos rojos, o sangrado que contribuyera a la muerte $u$ ocurriese en un sitio crítico) y el secundario la ocurrencia de eventos hemorrágicos y mayores sangrados menores clínicamente relevantes.

\section{Resultados principales}

Los resultados principales se resumen en la tabla 1.

Tabla 1: resultados clínicos durante el periodo activo (12 meses).

\begin{tabular}{l|c|c|c|c|c|c} 
& $\begin{array}{c}\text { Apixaban 2,5mg } \\
(\mathbf{N = 8 4 0})\end{array}$ & $\begin{array}{c}\text { Apixaban 5mg } \\
(\mathbf{N = 8 3 0})\end{array}$ & $\begin{array}{c}\text { Placebo } \\
(\mathbf{N = 8 2 9 )}\end{array}$ & $\begin{array}{c}\text { Apixaban 2,5 mg } \\
\text { vs. Placebo }\end{array}$ & $\begin{array}{c}\text { Apixaban 5 mg } \\
\text { vs. Placebo }\end{array}$ & $\begin{array}{c}\text { Apixaban 2,5 } \\
\text { vs. 5 mg }\end{array}$ \\
\cline { 2 - 7 } & Número (\%) & Número (\%) & Número (\%) & RR (IC 95\%) & RR (IC 95\%) & RR (IC 95\%) \\
\hline $\begin{array}{l}\text { TEV recurrente o muerte por cualquier } \\
\text { causas }\end{array}$ & $32(3,8)$ & $34(4,2)$ & $96(11,6)$ & $0,33(0,22$ a 0,48) & $0,36(0,25$ a 0,53$)$ & ND \\
\hline TEV recurrente o muerte por TEV & $14(1,7)$ & $14(1,7)$ & $73(8,8)$ & $0,19(0,11$ a 0,33$)$ & $0,20(0,11$ a 0,34$)$ & $0,97(0,46$ a 2,02$)$ \\
\hline Sangrado mayor & $2(0,2)$ & $1(0,1)$ & $4(0,5)$ & $0,49(0,09$ a 2,64$)$ & $0,25(0,03$ a 2,24$)$ & $1,93(0,18$ a 21,25) \\
\hline $\begin{array}{l}\text { Sangrado menor clínicamente } \\
\text { relevante }\end{array}$ & $25(3,0)$ & $34(4,2)$ & $19(2,3)$ & $1,29(0,72$ a 2,33$)$ & $1,82(1,05$ a 3,18$)$ & $0,71(0,43$ a 1,18$)$ \\
\hline
\end{tabular}

TEV: tromboembolismo venoso. ND: no disponible. §Los 13,20 y 19 pacientes pertenecientes al grupo apixaban 2,5mg, apixaban $5 \mathrm{mg}$ y placebo, respectivamente, que se perdieron del seguimiento fueron clasificados como si hubiesen presentado el resultado principal.

\section{Conclusiones}

En comparación con placebo, el apixaban en las dos dosis evaluadas

$(2,5 \mathrm{mg}$ y $5 \mathrm{mg})$ redujo el riesgo de recurrencia de eventos tromboembóli-

cos (fatales y no fatales), sin observarse diferencias significativas en las tasas de sangrado mayor.

Fuente de financiamiento: Bristol-Myers y Pfizer

\section{Comentario}

El apixaban, un inhibidor directo del factor $\mathrm{Xa}$, es uno de los nuevos anticoagulantes orales diseñados por la industria biomédica con el objetivo de administrar una dosis fija de medicación sin el requerimiento de monitoreo de laboratorio. En este estudio se compara la efectividad de dos dosis $(2,5 \mathrm{mg}$ y $5 \mathrm{mg}$, dos veces por día) de tratamiento extendido por un año versus placebo para evitar la recurrencia de TEV en pacientes que habían recibido entre 6 y 12 meses de anticoagulación por TEP o TVP confirmados. También se comparó la ocurrencia de eventos hemorrágicos en cada rama del estudio. El apixaban demostró ser más efectivo que el placebo en cualquiera de las dos dosis, con similar riesgo de sangrado que en el grupo placebo.

Varios estudios compararon el tratamiento extendido con otros nuevos anticoagulantes frente a warfarina o heparina de bajo peso molecular. El estudio RE-MEDY comparó dabigatran, warfarina y placebo, observándose que el dabigatran (inhibidor de la trombi- na) ${ }^{1}$ fue tan efectivo como la warfarina en la prevención del TEV recurrente y con menor riesgo de sangrado que ésta; mientras que ambas drogas fueron superiores al placebo pero con mayor riesgo de sangrado².

El dabigatran y el apixaban no han sido aprobados aún para el tratamiento a corto o largo plazo del TEV, pero si el rivaroxaban, droga que demostró ser más efectiva que el placebo $(82 \%$ reducción del riesgo relativo) aunque con mayor riesgo de sangrado ${ }^{3}$.

\section{Conclusiones del comentador}

El apixaban podría ser un recurso efectivo en la anticoagulación extendida de los pacientes para evitar la recurrencia de eventos tromboembólicos.

María Paula Cárdenas [ Sección Hematología. Servicio de Clínica Médica del Hospital Italiano de Buenos Aires.

maria.cardenas@ hospitalitaliano.org.ar]

Cárdenas MP. El apixaban fue efectivo en comparación con placebo en el tratamiento extendido del tromboembolismo venoso. Evid Act Pract Ambul. 2014:17(3).Jul-Sep. 83. Comentado de: Agnelli G, y col. Apixaban for Extended Treatment of Venous Thromboembolism. NEJM 2013;368:699708. PMID : 23216615.

\section{Referencias}

1. Garcia D, y col. The new anticoagulants. Blood 2010 Jan 7;115(1):15-20.

2. Schulman S, y col. Extended use of Dabigatran, Warfarin, or Placebo in venous thromboembolism. N Engl J Med 2013 2013; 368(8):709-18 3. Connors JM. Extended treatment of venous thromboembolism. NEJM 2013;368(8):767-69. 